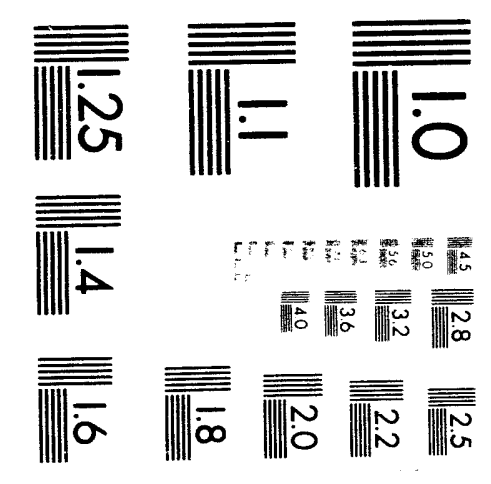



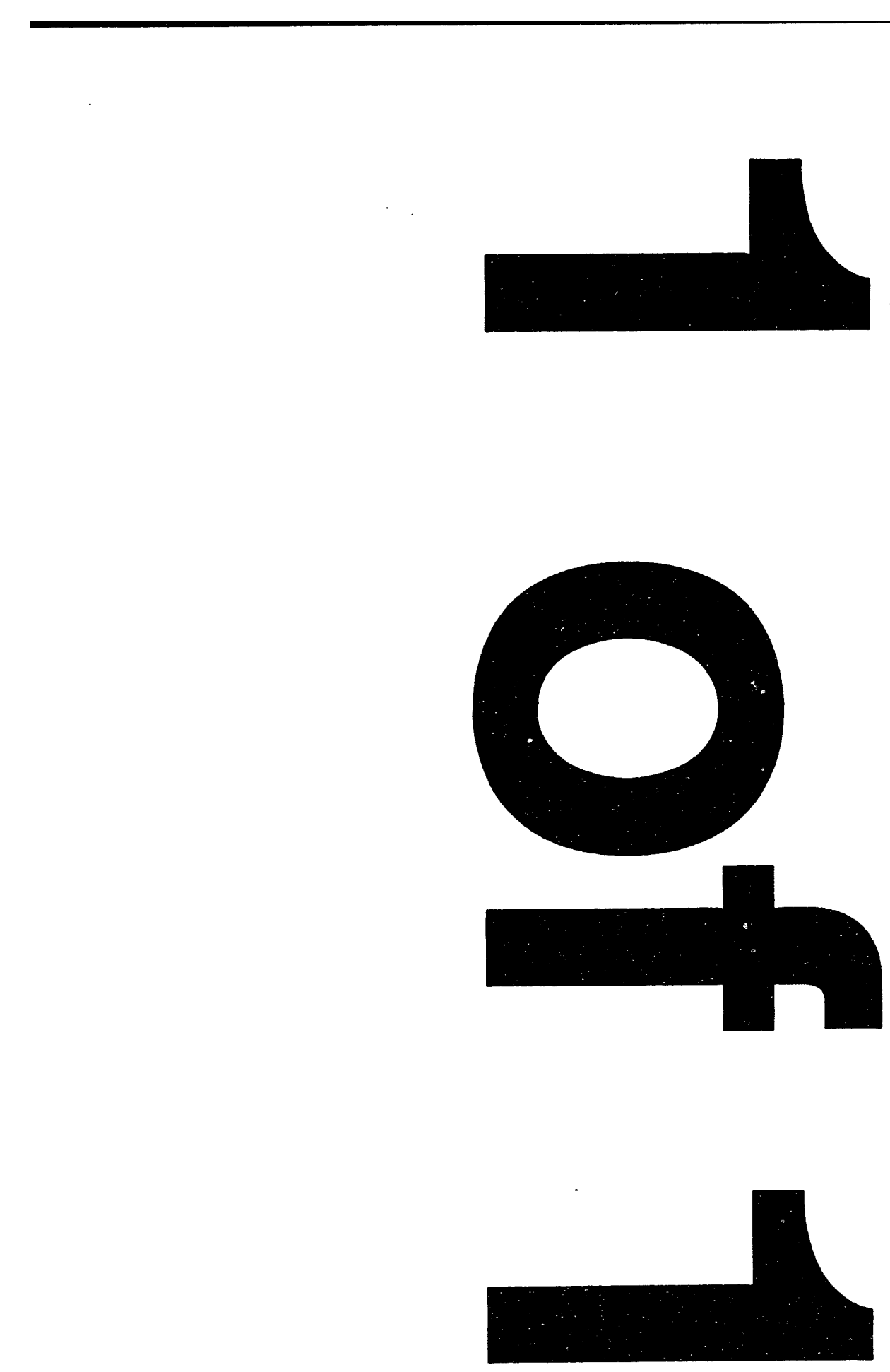
SESAME Equation of State Number 8020:

Polyetheretherketone (PEEK)

J. C. Boettger

J. D. Johnson 


\title{
SESAME Equation of State Number 8020: \\ Polyetheretherketone (PEEK)
}

by

\author{
J. C. Bocttger and J. D. Johnson
}

\begin{abstract}
A new SESAME equation of state (EOS) for the polymer polyetheretherketone (PEEK) has been generated using the computer program GRIZZLY. This new EOS has been added to the SESAME EOS library as material number 8020 .

A few general guidelines for estimating the thermodynamic parameters for polymers needed to generate an EOS with GRIZZLY are suggested.
\end{abstract}

\section{INTRODUC'TION}

The SESAME equation of state (EOS) library has been a valuable data resource for its many users since its inception. ${ }^{1}$ Currently, the library contains EOSs for roughly 150 materials with a wide range of physical characteristics; e.g., metals, insmintor ", liquids, rocks, etc. In recent years, one of the most significant areas of ga ow the the library has been polymers and polymer composites (materials produced by nuctimg some relatively hard substance inside a polymer matrix). For example, wa 59 AMP library already includes EOSs for silicone rubber, ${ }^{2}$ boron-filled silicone, ${ }^{3}$, phenolic refrasil, ${ }^{4}$ and polycarbonate, ${ }^{5}$ to name only a few. This interest in the EOSs of polymers and composites has been motivated by the wide range of properties 
exhibited hy these materials and the case vith which they can be fabricated in any desired shape.

Unfortunately, hy definition, polymers have exceptionally complicated molecular structures, which make their EOSs unusually difficult to model; see discussion on page 6. In addition, many of the newer polymers (and even some of the older ones) are poorly characterized experimentally. Thus, it is often impossible to find reliable thermodynamic data to be used as input to GRIZZLY, the locally developed computer program for calculating SESAME EOSs. It is particularly difficult to find meaningful data for the Debye temperature $\left(\Theta_{D}\right)$, the Gruneisen parameter $\left(\gamma_{0}\right)$, and the cohesive $\operatorname{encrgy}\left(E_{c}\right)$.

In this investigation, a SESAME EOS has been constructed for polyetherether- $\checkmark$ ketone (PEEK), a relatively new "high performance engineering" thermoplastic. In the course of developing the new EOS for PEEK, a simple pragmatic approach to estimating $\Theta_{D}, \gamma_{0}$, and $E_{c}$ for polymers has been developed based on experience accumulated during the past few years. Some basic guidelines for estimating the needed data and the general ideas underlying them will be discussed here in considerable detail. The new EOS for PEEK will be added to the SESAME library as material number 8020.

In the next section, the models and input parameters used to generate the EOS for material number 8020 are described in detail. The new SESAME EOS for PEEK is presented and discussed in Section III. 


\section{METHODOLOGY}

Most of the EOSs in the SESAME library are partitioned into three terms for the pressure $P$, the internal energy $E$, and the Helmholtz free energy $A$ :

$$
\begin{aligned}
& P(\rho, T)=P_{s}(\rho)+P_{n}(\rho, T)+P_{e}(\rho, T) \\
& E(\rho, T)=E_{s}(\rho)+E_{n}(\rho, T)+E_{e}(\rho, T) \\
& A(\rho, T)=A_{s}(\rho)+A_{n}(\rho, T)+A_{e}(\rho, T)
\end{aligned}
$$

where $\rho$ is the density and $T$ is the temperature. (In the SESAME library, discrete values of $\rho$ and $T$ are chosen to form a mesh on which $P, E$, and $A$ are stored.) The subscripts $s, n$, and $e$ denote the contributions from the static lattice (i.e., frozen nuclei) cold curve (zero temperature isotherm), the nuclear motion, and the thermal electronic excitations, respectively. It is thus possible to calculate (or update) each term independently using any desired model. Here, all three pieces of the EOS have been generated with GRIZZLY. ${ }^{6}$

\section{Electronic Contributions}

In GRIZZLY, the only model currently available for calculating the thermal electronic contributions is the Thomas-Fermi-Dirac (TFD) model. ${ }^{7}$ First, thermal electronic contributions were generated for each individual atom in PEEK. Those monatomic thermal electronic EOSs were then combined via additive volume mixing ${ }^{6}$ to form the total electronic contribution to the EOS. This part of the calculation requires the number fraction $\left(N_{i}\right)$, atomic number $\left(Z_{i}\right)$, and atomic mass $\left(A_{i}\right)$ for each species of 
atom. The nominal chemical formula for an isolated monomer of PEEK (composed of three henzene rings coupled by two oxygens and one $\mathrm{CO}$ ) is $\mathrm{C}_{19} \mathrm{H}_{12} \mathrm{O}_{3}{ }^{8}$ It is trivial to calculate the needed values of $N_{i}$ from the monomer formula; see Table 1. Table 1 also lists the values of $A_{i}$ and $Z_{i}$ for each constituent atom in PEEK. ${ }^{9}$

Table 1: Atomic Composition Of PEEK

\begin{tabular}{|l|c|c|c|}
\hline Atom & $N_{i}$ & $A_{i}$ & $Z_{i}$ \\
\hline & & & 6.0 \\
$\mathrm{C}$ & 0.5588 & 12.01 & 1.0 \\
$\mathrm{H}$ & 0.3529 & 1.008 & 8.0 \\
\hline
\end{tabular}

\section{Nuclear Contributions}

The thermal nuclear contributions for PEEK were obtained with the JDJNUC nuclear model ${ }^{10}$ in GRIZZLY. In this model, the material is treated as a Debye solid at low temperatures and as an ideal gas at high temperatures. The JDJNUC model smonthly switches hetween these two limits for temperatures near the melt line, which is obtained from the Lindemann law. At the melting point on each isotherm, the JDJNUC model huilds in a small two-phase region to simulate the melting transition. In addition to the data in Tahle 1, this part of the calculation requires an ambient value for the melt ternperature $\left(T_{m}\right)$ and some analytical form for the Gruneisen parameter as a function of density $[\gamma(\rho)]$. The Dehye temperature $\left(\Theta_{D}\right)$ is computed from $T_{m}$ using an empirical relationship that is based on the hehavior of pure elements.

Because PEEK is a thermoplastic, it has a well-defined melting temperature (6.30) 
$\mathrm{K})^{8}$ to be used as input to GRIZZLY. This value for $T_{m}$ produces a reasonable Debye temperature of 560) K. However, many polymers are not so well behaved. In general, thermoset polymers (e.g., phenolic and epoxy) do not have a clearly defined melting transition. Instead, as the temperature is increased, thermoset polymers will be only partially melted and/or vaporized until very high temperatures are achieved. For these polymers, it makes more sense to estimate $\Theta_{D}$ and then back out an effective $T_{m}$ as was done in Ref. 3. Even this approach is not straightforward since it is generally possible to define two values of $\Theta_{D}$ for any given polymer; one related to the intrachain phonon modes and the other (smaller value) associated with the interchain phonon modes. ${ }^{2}$ For the purpose of forming SESAME EOSs the higher value of $\Theta_{D}$ should be used, since it will be applicable to temperatures above ambient. In the absence of experimental data for both $T_{m}$ and $\Theta_{D}$, it is suggested here that for a thermoplastic a melt temperature in the range of about $400 \mathrm{~K}$ to $700 \mathrm{~K}$ should be used, while for a thermoset a melt temperature on the order of $1(000 \mathrm{~K}$ or greater would be more appropriate.

A generalized version of the standard CHART-D mode ${ }^{11}$ has been used to describe $\gamma(\rho)$; the IGRUN $=7$ option in GRIZZLY. ${ }^{12}$ In this formulation, the Gruneisen parameter is expressed as a quadratic function of $\rho$ in the expanded region and as a quadratic function of $1 / \rho$ in the compressed region. Four items of input are required to completely specify $\gamma(\rho)$ : ambient values for $\gamma(\rho)$ and its derivative with respect to $\ln \rho\left[\gamma_{0}\right.$ and $\left.d \gamma / d(\ln \rho)\right]$, and its asymptotic values $\gamma(0)$ and $\gamma(\infty)$. For $\gamma(\infty)$, the default value of $2 / 3$ has been used here. For the ambient logarithmic derivative, a 
fairly small negative value (-(0.(5) was selected to ensure reasonably smooth thermal nuclear isotherms near $\rho_{0}$. The two remaining input parameters, $\gamma_{0}$ and $\gamma(0)$, are somewhat more difficult to estimate.

Within GRIZZLY, $\gamma$ is assumed to be independent of $T$; a reasonable approximation for most classes of materials. However, for polymers, the Gruneisen parameter is known to be highly temperature-dependent because of the large difference between the interchain and intrachain phonon modes. ${ }^{13}$ At low temperatures, the soft interchain modes dominate and $\gamma$ is quite large; on the order of $5 .{ }^{13}$ As the temperature increases, the interchain modes quickly saturate and the stiffer intrachain modes dominate. As a result, $\gamma$ rapidly drops to values which are in the range $0.2<\gamma<1.3$. (Here the limits have been estimated by silicone rubber ${ }^{2}$ and phenolic. ${ }^{4}$ ) The high temperature intrachain value scems most appropriate for the present purpose. As an additional complication, no experimental value of $\gamma_{0}$ is available for PEEK. Here, the value of $\gamma_{0}$ has heen estimated by requiring the final EOS to reproduce the experimental thermal expansion coefficient; $\alpha(400) \mathrm{K}) \approx 4 \rightarrow 6 \times 10^{-5} \mathrm{~K}^{-1}$. The final value of $\gamma_{0}$ used here (0.33) yielded $\alpha(40) K) \approx 6 \times 10^{-5} K^{-1}$. Since $\alpha$ is generally known even for the newer polymers, this procedure should be widely applicable.

The final input parameter needed to determine $\gamma(\rho)$ is the low-density limit $\gamma(0)$. If $\gamma(0)$ is chosen to be significantly smaller than $\gamma_{0}$, the nuclear isotherms cross; a clearly unphysical result. On the other hand, if $\gamma(0)$ is chosen to be significantly larger than $\gamma_{0}$, the nuclear isotherms exhibit a significant amount of structure near the ambient density, which is hard to justify on physical grounds. To avoid both of these difficulties, 
an intermediate value was chosen; $\gamma(0)=2 / 3$. Similar considerations should apply to other polymers that have small values of $\gamma_{0}$.

\section{Cold Curve}

The static lattice cold curve for modest compressions $(\leq 2.0)$ was calculated by removing thermal contributions from a Hugoniot that was read in as a table of particle and shock velocities $\left(U_{p}, U_{s}\right)$ chosen to give a reasonable fit to the available Hugoniot and sound speed data; ${ }^{14}$ see Table 2 . For high compressions, the cold curve was required to smoothly extrapolate to a mixed TFD cold curve. This method of calculalion ensures that the compressed EOS will reproduce the experimental Hugoniot and also have the correct asymptotic behavior. In the expanded region $\left(\rho<\rho_{0}\right)$, the cold curve was forced to have a generalized Lennard-Jones form ${ }^{6}$ that was constrained to smoothly connect with the compressed portion of the cold curve and have the correct cohesive energy $\left(E_{c}\right)$. In addition to the parameters already mentioned, the expanded cold curve calculation requires a parameter FACLJ ${ }^{3}$ (here 0.2 ), which controls the shape of the expanded cold curve.

Table 2: Input Hugoniot Points For PEEK

\begin{tabular}{|l|c|c|c|}
\hline$U_{p}(\mathrm{~km} / \mathrm{s})$ & $U_{s}(\mathrm{~km} / \mathrm{s})$ & $U_{p}(\mathrm{~km} / \mathrm{s})$ & $U_{s}(\mathrm{~km} / \mathrm{s})$ \\
\hline & & & \\
$0 .(0)$ & 2.30() & 3.35 & 6.758 \\
1.21 & 4.447 & 5.00 & 9.018 \\
2.69 & 6.517 & & \\
\hline
\end{tabular}


The only input parameter which remains to be determined is the cohesive energy $\left(E_{c}\right)$. Once again, this parameter is difficult to select for polymers, due to their complicated molecular structure. In the absolute sense, there is no ambiguity about determining $E_{c}$. It is simply the energy required to divide the system into a collection of isolated atoms; typically $100 \mathrm{kcal} / \mathrm{avg}$-atom-mole for a hydrocarbon. However, using this rather large value for $E_{c}$ generally will result in a predicted critical temperature $\left(T_{c}\right)$ on the order of 3000$) \rightarrow 7000 \mathrm{~K}$, a value which is probably too large for many polymers, depending on precisely how they dissociate at high temperatures.

For a thermoplastic such as PEEK, it is likely that the polymer chains will break up into a collection of benzene molecules (and/or other volatiles) at a temperature on the order of $10(0) \rightarrow 30(0) \mathrm{K}$. At much higher temperatures (roughly 5000$) \mathrm{K}$ or higher) the individual molecules will decompose into individual atoms. In this case, it will be more reasonable to approximate $E_{c}$ with the energy needed for the initial dissociation into molecules rather than using the absolute cohesive energy. Thus, $E_{c}$ can be adusted to obtain a realistic $T_{c}$; say $2 \rightarrow 4 \times T_{m}$. For PEEK, $E_{c}=35 \mathrm{kcal} / \mathrm{avg}$-atom-mole was used, resulting in $T_{c}=2050 \mathrm{~K}\left(\approx 3.3 \times T_{m}\right)$.

In contrast to the thermoplastic polymers, thermoset polymers generally have strong crosslinking between the molecular chains and may remain solid at very high temperatures, although some volatiles will be driven off at lower temperatures. The solid residue left hehind will he primarily composed of carhon, for which $\left.T_{c}=8200\right)$ K. ${ }^{15}$ Although a thermoset polymer will not have a well-defined $T_{m}$, it is clear that its $T_{c}$ should be significantly higher than for a thermoplastic. It is suggested here that the 
absolute value of $E_{c}$ should be used for a thermoset, unless an unrealistically large value of $T_{c}$ results.

\section{RESULTS}

Figure 1 compares the present theoretical Hugn:iint for PEEK with experimental data from Ref. 14. There are two important features apparent in both the experimental data and the theoretical line. First, there is a phase transition in the region $2.7<U_{p}<3.2 \mathrm{~km} / \mathrm{s}$. This phase transition is characteristic of many polymers and may be due to pressure-induced crosslinking between the chains. ${ }^{16}$ Second, as $U_{p}$ approaches zero, $U_{s}$ drops more rapidly than would be expected based on the nearly linear behavior exhibited in the range $1.0 \mathrm{~km} / \mathrm{s}<U_{p}<2.7 \mathrm{~km} / \mathrm{s}$. Again, this result is fairly standard for polymers (especially soft ones) and is due to the large difference between the interchain and intrachain binding. ${ }^{16}$ As the interchain separation is decreased under pressure, the bulk modulus and $U_{s}$ both increase rapidly until the interchain spacing becomes comparable to the intrachain spacing, at which point the Hugoniot becomes linear. In general, the new EOS for PEEK provides a good fit to the experimental Hugoniot data.

Figure 2 shows a selected group of $P$ vs. $\rho$ isotherms from the new SESAME EOS for PEEK. In general, the theoretical isotherms reveal no pathologies in the new EOS. The effect of the solid-solid phase transition (discussed above) on the isotherms can be seen for $\rho \approx 2.5 \mathrm{gm} / \mathrm{cm}^{3}$. One of the isotherms shown in Fig. 2 has $T=T_{c}=$ $2050 \mathrm{~K}$. Although it is not apparant in Fig. 2, the new EOS for PEEK also reproduces 
the experimental thermal expansion coefficient, as was discussed above.

Overall the new EOS for PEEK should be reliable for all applications which involve shock loading; or other processes that lie near the principal Hugoniot. This new EOS will be added to the SESAME library as material number 8020 . 


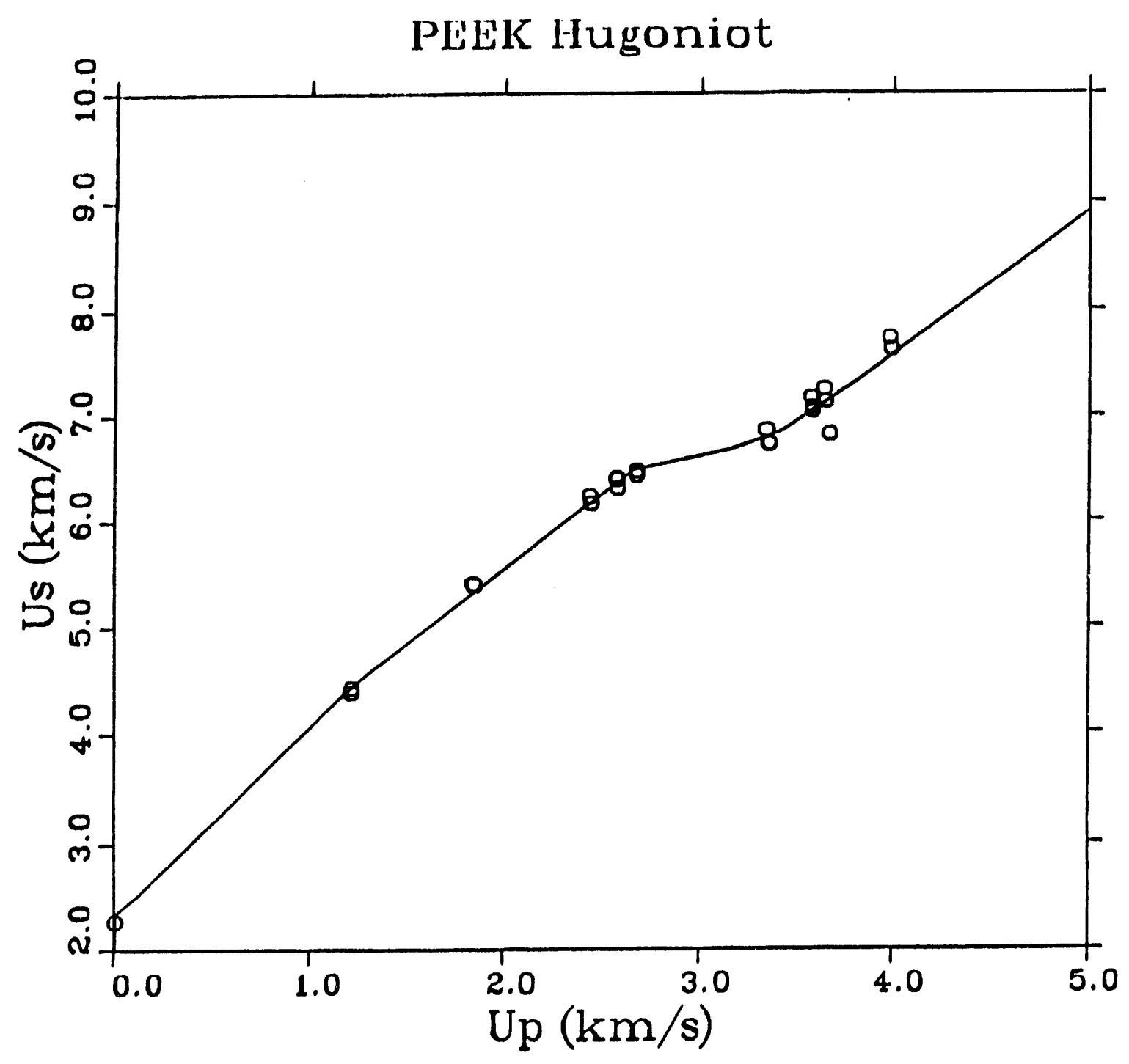

Figure 1. The theoretical Hugoniot for PEEK is compared with Hugoniot data from Ref. 14. 


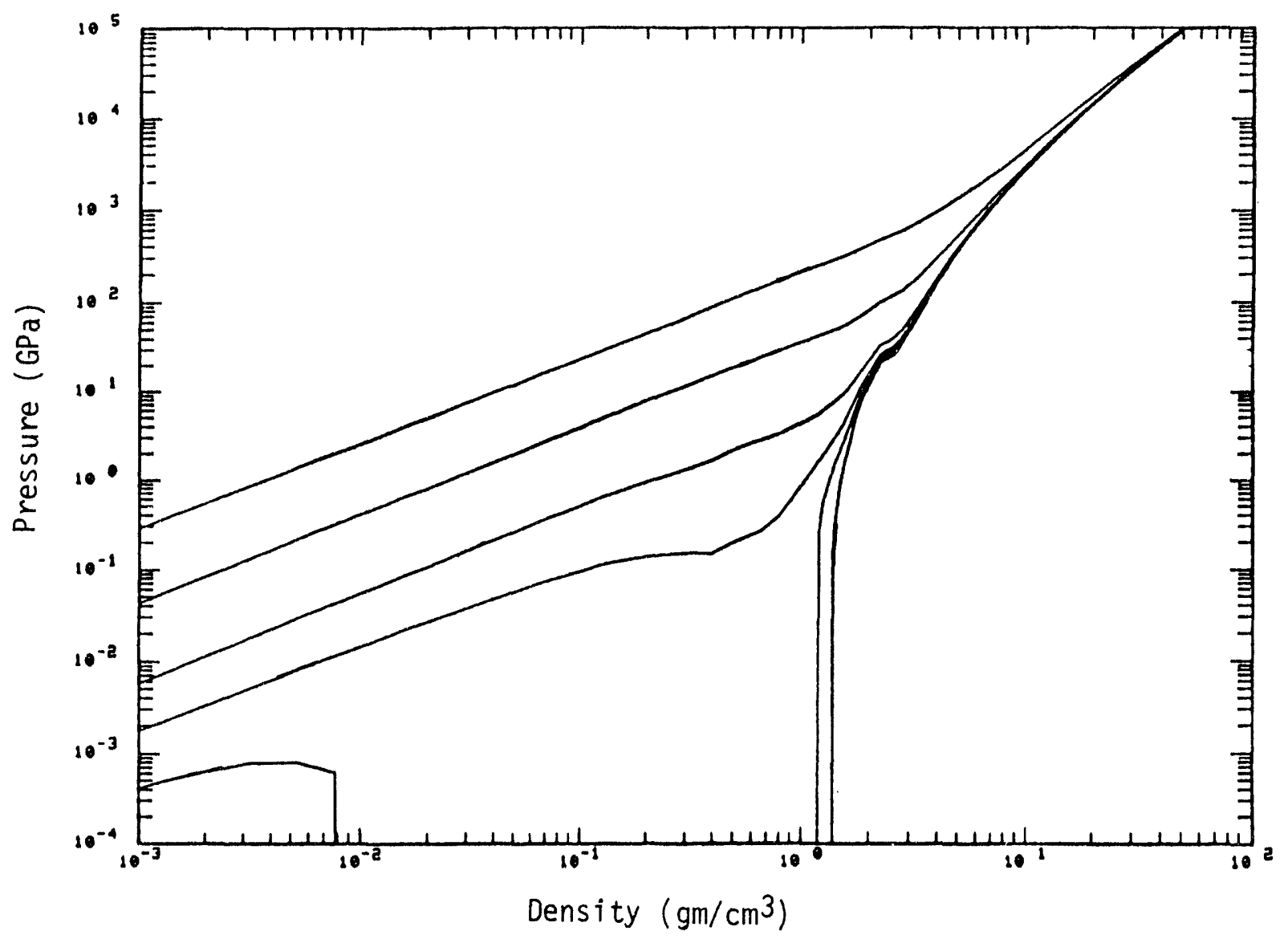

Figure 2. Selected isotherms from the theoretical SESAME EOS for PEEK. The temperatures included are $0 \mathrm{~K}, 1000 \mathrm{~K}, 2050 \mathrm{~K}, 5000 \mathrm{~K}, 25000 \mathrm{~K}$, and $100000 \mathrm{~K}$. 


\section{REFERENCES}

1. S. P. Lyon and J. D. Johnson, editors, "SESAME: The Los Alamos National Laboratory Equation Of State Database," Los Alamos National Laboratory Document LA-UR-92-3407 (October 1992).

2. F. Dowell, "A simple EOS for the Silicone Rubher Sylgard 184," Los Alamos National Laboratory Report LA-10164-MS (August 1984).

3. J. C. Boettger, "SESAME Equation Of State Number 8()10: Boron Loaded Silicone Potting Material," Los Alamos National Laboratory Report LA-12541-MS (May 1993).

4. J. C. Boettger, "SESAME Equation of State for a Polymer Composite: Phenolic Refrasil," Los Alamos National Lahoratory Report LA-11512-MS (March 1989).

5. J. C. Boettger, "SESAME Equation Of State Number 7740: Polycarbonate," Los Alamos National Laboratory Report LA-12120-MS (May 1991).

6. Joseph Abdallah, Jr., "User's Manual for GRIZZLY," Los Alamos National Laboratory report LA-10244-M (September 1984).

7. R. D. Cowan and J. Ashkin, Phys. Rev. 105, 144 (1957).

8. C. Sandoval, personal communication (May 1993).

9. N. W. Asheroft and N. D. Mermin, Solid State Physics (Holt, Rinehart, and Winston, New York, 1976).

10. J. D. Johnson, "A Generic Model For The Ionic Contribution To The Equation Of State," Los Alamos National Laboratory Report LA-12003-MS (January 1991). 
11. S. L. Thompson and H. S. Lawson, "Improvements to the CHART-D RadiationHydrodynamics Code III; Revised Analytic Equations of State," Sandia Laboratories report SC-RR-710714 (1972).

12. S. P. Lyon, "Addenda for GRIZZLY Table I-1," unpublished report obtainable from Group T-1 at Los Alamos National Laboratory.

13. Y. K. Godovsky, Thermophysical Properties Of Polymers (Springer-Verlag, New York, 1992).

14. B. Olinger, J. Fritz, and C. E. Morris, "Equations Of State For PEEK, Epon 828, and Carbon Fiber - Epon Composite," unpublished preliminary report, Los Alamos National Laboratory (March 1993).

15. R. W. Ohse and H. von Tippelskirch, High T. - High P. 9, 367 (1977).

16. W. J. Carter, S. P. Marsh, and R. G. McQueen, "Hugoniot Equation of State of Polymers," Los Alamos Scientific Laboratory document LA-UR-77-2062 (1977). 

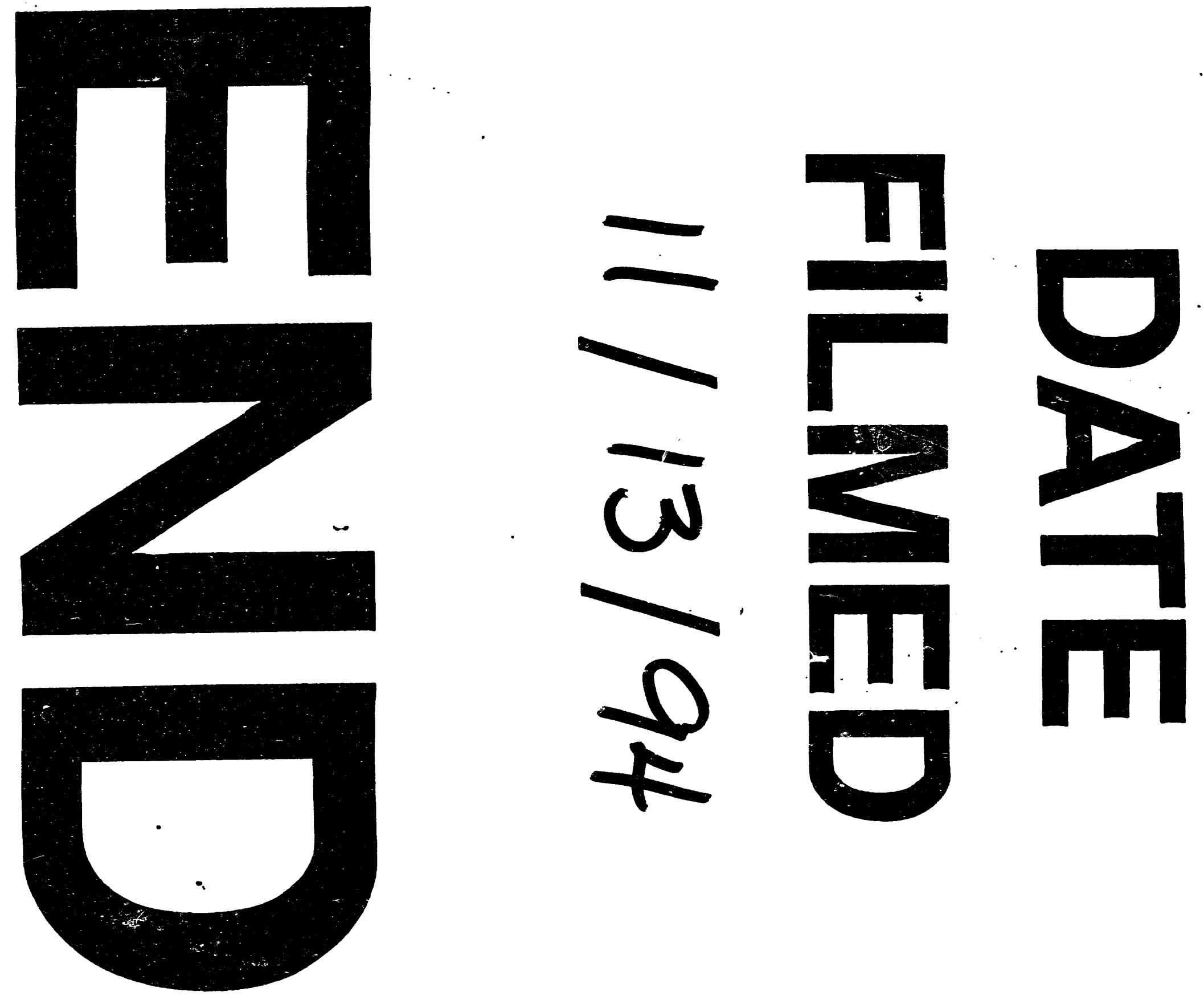
